\title{
Long term follow-up of a phase II trial of multimodal therapy given in a "sandwich" method for stage III, IV, and recurrent endometrial cancer
}

Michelle Glasgow ${ }^{1}$, Rachel Isaksson Vogel ${ }^{1,2}$, Jennifer Burgart ${ }^{3}$, Peter Argenta ${ }^{1}$, Kathryn Dusenbery ${ }^{4}$ and Melissa A. Geller ${ }^{1,5^{*}}$

\begin{abstract}
Background: Our objective was to determine if previously reported overall survival (OS) and progression-free survival (PFS) rates are maintained long term following multimodal therapy for advanced and recurrent endometrial cancer and to assess the lymphedema rates associated with this therapy.

Methods: Women with advanced-stage or recurrent endometrial cancer were recruited between 9/2004 and 6/2009 to our previously published Phase II trial. Patients received intravenous docetaxel $(75 \mathrm{mg} / \mathrm{m} 2)$ and carboplatin (AUC =6) every 3 weeks for 3 cycles before and after radiation therapy. Patient outcomes were updated in July 2014. Data abstracted included presence of lymphedema, disease progression, and death. OS and PFS estimates at 5 years were calculated using Kaplan-Meier methods.

Results: Of the 41 patients enrolled, 10 (24\%) had stage IIIA and 21 (51\%) had stage IIIC disease; 32 (78 \%) had endometrioid histology; and 35 (85\%) completed the protocol. With a median follow-up of 5 years, 15 of 41 patients have died. The Kaplan-Meier estimate and $95 \% \mathrm{Cl}$ for OS at 5 years was $70 \%$ (53-82 \%). Excluding the two patients with recurrent disease at enrollment, 15 of 39 patients progressed or died during follow-up. The Kaplan-Meier estimate and $95 \% \mathrm{Cl}$ for PFS at 5 years was $66 \%$ (48-78\%). Fifteen patients (37\%) had medical record documentation of lymphedema following treatment.

Conclusions: After additional follow-up, OS and PFS estimates remain high and in-field recurrences low following "sandwich" therapy. The "sandwich" method remains efficacious for women with stage III-IV or recurrent endometrial cancer.
\end{abstract}

Keywords: Endometrial cancer, Sandwich therapy, Lymphedema

\footnotetext{
*Correspondence: gelle005@umn.edu

Preliminary data from this study were presented at the Annual Meeting of the Society of Gynecologic Oncologists in March 2015.

'Department of Obstetrics, Gynecology and Women's Health, Division of Gynecologic Oncology, University of Minnesota, Minneapolis, MN, USA

${ }^{5}$ University of Minnesota, MMC 395, 420 Delaware St. SE, Minneapolis, MN

55445, USA

Full list of author information is available at the end of the article
} 


\section{Background}

Endometrial cancer is the most common gynecologic malignancy in the United States with an estimated 52,630 new cases occurring in 2014 [1]. While most patients present with early stage disease and can be cured with surgery alone, survival is poor for those with advanced disease [2]. Adjuvant chemotherapy has been shown to control distant recurrence while adjuvant radiation has demonstrated control of local disease [3]. Several studies in recent years have shown that the combination of these two treatment modalities may be the most promising option for patients with advanced disease.

In 2011, we presented the results of a Phase II trial examining the use of carboplatin and docetaxel followed by radiation treatment and additional chemotherapy, according to the "sandwich" method in women with advanced stage or recurrent endometrial cancer [4]. With a median follow up of 28 months of 41 evaluable patients, the Kaplan-Meier (KM) estimates for overall survival (OS) at 1 year were $95 \%$, at 3 years $90 \%$, and at 5 years $71 \%$; the KM estimates for progression-free survival (PFS) at 1 year were $87 \%$, at 3 years $71 \%$, and at 5 years $64 \%$.

Several others have published retrospective and prospective studies supporting our conclusions that the "sandwich" method for the treatment of advanced stage endometrial cancer is effective and well tolerated (Table 1). Abaid et al. [5] reported that among 32 patients with advanced stage endometrial cancer (or early stage with high risk features, such as high grade and presence of lymphovascular space invasion) treated with the "sandwich" approach, PFS was
$84 \%$, with a mean duration of follow-up of 18.9 months. Einstein et al. [6] examined the use of the "sandwich" method in 72 patients with both early and advanced stage uterine papillary serous carcinoma in a prospective Phase II trial; they estimated 3-year OS at $84 \%$ for patients with early stage disease and $50 \%$ for patients with advanced stage disease. In both of these studies, the rates of treatment completion were high (>90\%) and reported toxicity profiles acceptable. The hematologic and non-hematologic toxicities were mostly self-limited. However, the retrospective nature of these studies and their limited follow up prohibited a comprehensive assessment of long term treatment toxicities.

While these studies support the effectiveness of the "sandwich" method, toxicities do exist. Dogan et al. [7] examined acute toxicity following the "sandwich" approach in 25 women with stage IIIC endometrial cancer and compared their toxicities with those of women of the same stage who received chemotherapy followed by radiation alone. These authors found higher toxicity rates with more required dose reductions and treatment breaks, especially when pelvic and para-aortic lymph nodes were included in the radiation field. Published studies to date have not examined the long-term toxicities associated with the "sandwich" method. One of the most concerning toxicities is that of lymphedema, given the physical discomfort, pain, reduction in mobility, and negative impact on body image. [8-11]. Beesley et al. [9] recently reported the cumulative incidence of lymphedema after treatment for endometrial cancer in the Australian National

Table 1 Reported progression-free and overall survival for endometrial cancer patients treated with "sandwich" chemotherapy and radiation therapy

\begin{tabular}{|c|c|c|c|c|c|c|c|c|}
\hline \multirow[b]{2}{*}{ Lead Author, Year } & \multicolumn{5}{|c|}{ Overall survival } & \multicolumn{3}{|c|}{ Progression-free survival } \\
\hline & $\mathrm{N}$ & $\begin{array}{l}\text { UPSC/clear cell/ } \\
\text { mixed }\end{array}$ & Deaths & 3-years OS & $\begin{array}{l}\text { Median follow-up } \\
\text { (months) }\end{array}$ & $\mathrm{N}$ & $\begin{array}{l}\text { Recur, progression } \\
\text { or death }(\mathrm{N})\end{array}$ & 3-years PFS \\
\hline Gehrig, 2004 [25] & 9 & $100 \% / 0 \% / 0 \%$ & 0 & $100 \%$ & 38 & N/A & 1 & N/A \\
\hline aLupe, 2007 [26] & 33 & $33 \% / 9 \% / 15 \%$ & 13 & $55 \%$ (2-years) & 21 & 33 & 14 & $55 \%$ (2-years) \\
\hline Secord, 2007 [27] & 51 & N/A & 5 & $91 \%$ & 36 & 51 & 13 & $69 \%$ \\
\hline${ }^{\mathrm{a}}$ Fields, 2008 [28] & 30 & $100 \% / 0 \% / 0 \%$ & 10 & $52 \%$ & N/A & 29 & 12 & $54 \%$ \\
\hline aLupe, 2009 [16] & 43 & $35 \% / 7 \% / 14 \%$ & 14 & $68 \%$ & 30 & 41 & 35 & $53 \%$ \\
\hline Secord, 2009 [29] & 45 & $13 \% / 4 \% / 29 \%$ & 7 & $88 \%$ & 36 & 45 & 11 & $69 \%$ \\
\hline Geller, 2010 [17] & 23 & $52 \% / 4 \% / 0 \%$ & 3 & $88 \%$ & 44 & 23 & 5 & $80 \%$ \\
\hline${ }^{\mathrm{a}}$ Geller, 2011 [4] & 41 & $9 \% / N A / 2 \%$ & 7 & $90 \%$ & 28 & 39 & 11 & $71 \%$ \\
\hline Abaid, 2012 [5] & 32 & $13 \% / 9 \% / 9 \%$ & 3 & N/A & 19 & 8 & 8 & $84 \%$ \\
\hline aEinstein, 2012 [6] & & $100 \% / 0 \% 0 \%$ & & $\begin{array}{l}84 \% \text { (early stage); } \\
50 \% \text { (advanced stage) }\end{array}$ & 9 & 20 & 20 & N/A \\
\hline Dogan, 2013 [7] & 11 & $18 \% / 18 \% / 0 \%$ & 0 & N/A & 18 & 1 & 5 & N/A \\
\hline Lan, 2013 [30] & 35 & $\mathrm{~N} / \mathrm{A}$ & 4 & $82 \%$ & 36 & 35 & 9 & $62 \%$ \\
\hline $\begin{array}{l}\text { aGeller, } 2011 \text { - } \\
\text { updated }\end{array}$ & 41 & $9 \% / N A / 2 \%$ & 15 & $75 \%$ & 60 & 39 & 15 & $71 \%$ \\
\hline
\end{tabular}

UPSC uterine papillary serous carcinoma, PFS progression-free survival

${ }^{\mathrm{a}}$ Prospective study 
Endometrial Cancer Study to be $13 \%$, consistent with previous reports which documented incidence rates between 1 and $18 \%$.

The primary aim of our current study is to present updated estimates of OS and PFS among women with advanced stage endometrial cancer who participated in our previously published Phase II trial [4]. Although important to review long term toxicity data such as gastrointestinal or genitourinary side effects, the symptoms associated with these side effects are often difficult to ascertain from a retrospective chart review. In this review, we focused on development of lymphedema instead of other long-term toxicities given few studies have reported lymphedema rates following multi-modality therapy.

\section{Methods}

\section{Patient selection}

Detailed methods for this Phase II trial are provided elsewhere [4]. Briefly, women with newly diagnosed advanced stage endometrial cancer (e.g. Stage IIIA-IVB according to the International Federation of Gynecology (FIGO) 1988 staging classification) or recurrent disease were recruited from the University of Minnesota Gynecologic Cancer Clinic and Methodist Hospital between 9/2004 and 6/2009. Patients were ineligible if they had received previous pelvic radiation or chemotherapy. Informed consent was obtained for all study participants for the original Phase II trial and University of Minnesota IRB approval (IRB\#1406M51501) was granted for the follow-up medical chart review.

\section{Treatment regimen}

Patients received 3 cycles (once weekly for 3 weeks) of IV docetaxel at $75 \mathrm{mg} / \mathrm{m}^{2}$ administered over $60 \mathrm{~min}$ followed by carboplatin area under the curve (AUC) of 6 administered over $30 \mathrm{~min}$, prior to radiation therapy. Radiotherapy was initiated within 4 weeks of the third cycle of chemotherapy and was delivered using a 4-field technique to the pelvis. All patients received pelvic irradiation; the fields were expanded to encompass the para-aortic nodal chains for those with positive para-aortic nodes. The total dose to the pelvic isocenter was $45.5 \mathrm{~Gy}$ and to the center of the paraaortic nodal tissue between 43 and 45 Gy. HDR brachytherapy vaginal cuff boost was delivered for vaginal extension, cervical involvement, lower uterine segment involvement, parametrial extension or at the discretion of the treating radiation oncologist. Following radiation, three identical cycles of chemotherapy were initiated within 6 weeks of completing radiotherapy.

\section{Patient follow-up}

Patients were evaluated before every chemotherapy cycle and weekly during radiation therapy. Adverse events were assessed and reported. After completion of treatment, participants underwent disease assessment with imaging 4-6 weeks following their final chemotherapy. Patients were evaluated for disease progression and recurrence every 3 months for 2 years and then every 6 months thereafter for an additional 3 years. Symptoms or exam findings concerning for disease progression and/or recurrence were confirmed with imaging and biopsy. Recurrences were defined as local if they occurred within the radiation field. Initial results were reported in 2011. Following approval from the University of Minnesota's Institutional Review Board, a retrospective medical chart review was conducted May-July 2014 to obtain updated follow-up on all participants in this trial. Clinical data abstracted included: reported lymphedema (defined as any documentation of patient-reported lymphedema confirmed by physical exam following treatment), date of disease progression, site of recurrence and additional treatment, and date of death or date of last follow-up for those last known to be alive.

\section{Statistical methods}

Patient demographic and clinical data were summarized and presented as frequencies (number, percent) or means \pm standard deviations as appropriate unless otherwise noted. OS was calculated from date of study enrollment to death or was censored at date of last contact for patients still alive. PFS was calculated from study enrollment date to the date of first known progression or death or was censored at date of last contact for patients still alive; two patients with recurrent disease at the time of study entry were excluded from the analysis of this outcome. KaplanMeier estimates and $95 \%$ confidence intervals (CI) at 3 and 5 years are reported [12]. Median OS and PFS are provided as well, however there were an insufficient number of deaths to accurately estimate the $95 \%$ CIs. The rate of lymphedema was calculated as the proportion of participants in the study with documented lymphedema.

\section{Results}

Forty-two patients enrolled in this trial between July 2004 and July 2009, however, ultimately 41 patients were evaluable as one patient withdrew prior to receiving any treatment. Table 2 describes the demographic and clinical characteristics of the study population. Of this group, ten patients had Stage IIIA disease, 21 had Stage IIIC disease, 1 had Stage IVA disease and 7 had Stage IV disease. The mean age at study entry was $59.0 \pm 11.5$ years, the majority had Stage IIIC endometrioid adenocarcinoma (51.2\%), and grades 2 and 3 histology were most common (41.5\% each). Other histologies included serous (9.8\%), mucinous (2.4\%), adenosquamous (7.3\%), and mixed serous and endometrioid (2.4\%). Two patients were treated at disease recurrence; the initial stages for these patients were Stage IC and IIA. Thirty-five patients completed the protocol as 
Table 2 Demographic and Clinical Data for all Participants ( $N=41)$

\begin{tabular}{|c|c|c|}
\hline & Number & Percent (\%) \\
\hline Age, years, mean (SD) & 41 & $59.0(11.5)$ \\
\hline \multicolumn{3}{|l|}{ Race } \\
\hline White & 35 & 85.4 \\
\hline Other & 5 & 12.2 \\
\hline Missing & 1 & 2.4 \\
\hline \multicolumn{3}{|l|}{ Disease Status } \\
\hline Primary & 39 & 95.1 \\
\hline Recurrence & 2 & 4.9 \\
\hline \multicolumn{3}{|l|}{ Stage } \\
\hline IC (recurrence) & 1 & 2.4 \\
\hline IIA (recurrence) & 1 & 2.4 \\
\hline$\| \mathrm{A}$ & 10 & 24.4 \\
\hline$\| I C$ & 21 & 51.2 \\
\hline IVA & 1 & 2.4 \\
\hline IVB & 7 & 17 \\
\hline \multicolumn{3}{|l|}{ Grade } \\
\hline 1 & 7 & 17.1 \\
\hline 2 & 17 & 41.5 \\
\hline 3 & 17 & 41.5 \\
\hline \multicolumn{3}{|l|}{ Histology } \\
\hline Endometrioid & 32 & 78.1 \\
\hline Serous & 4 & 9.8 \\
\hline Mucinous & 1 & 2.4 \\
\hline Adeno Squamous & 3 & 7.3 \\
\hline Endometrioid + Serous & 1 & 2.4 \\
\hline \multicolumn{3}{|l|}{ Surgery Type } \\
\hline Open & 32 & 84.2 \\
\hline Minimally Invasive & 6 & 15.8 \\
\hline Missing & 3 & \\
\hline \multicolumn{3}{|l|}{ Extent of lymphadenectomy } \\
\hline Any lymphadenectomy & 36 & 92.3 \\
\hline Pelvic lymphadenectomy & 5 & 13.9 \\
\hline Para-aortic lymphadenectomy & 1 & 2.8 \\
\hline Both & 30 & 83.3 \\
\hline
\end{tabular}

prescribed. Two participants were taken off protocol due to disease progression, one due to liver toxicity and three withdrew at their request.

Of the 39 patients with newly diagnosed disease, 36 patients had lymph nodes removed; five patients underwent pelvic lymphadenectomy alone, one patient underwent a para-aortic lymphadenectomy alone and 30 patients underwent both pelvic and para-aortic lymphadenectomy. The median number of pelvic lymph nodes removed was 17 (range: 0-32); the median number of para-aortic lymph nodes removed was 6 (range: $0-22$ ). Twenty-six patients received pelvic radiation only, while eight patients received extended-field radiation. Two received incomplete radiation; one due to disease progression and one at patient request. The median dose of external beam pelvic radiation was 45.5 Gy (range $=21-60 \mathrm{~Gy}$ ) in 25 fractions. The median length of time for completion of radiation was 38 days (15-55 days). Twenty-five patients were administered brachytherapy at a dose of 7 Gy in a single fraction to the proximal $4 \mathrm{~cm}$ of vagina at a depth of $0.5 \mathrm{~cm}$ and four patients received brachytherapy at a dose of 18 Gy in 3 fractions of $6 \mathrm{~Gy}$, each prescribed to the proximal $4 \mathrm{~cm}$ of the vaginal surface.

Updated data resulted in patients being followed for a median of 5 years (range: 0.5-9.6 years). One patient was lost to follow-up after treatment completion due to moving out of the country. There have been eight additional deaths since the initial report. Of the 41 patients enrolled, 15 patients died since the start of treatment (Fig. 1). The Kaplan-Meier estimate and $95 \% \mathrm{CI}$ for OS at 3 years was $75 \%$ (59-86\%) and 5 years was $70 \%$ (53-82\%); estimated median OS was 8.2 years.

After excluding the two patients who enrolled at recurrence, 15 of the 39 patients progressed or died during follow-up (Fig. 2). The Kaplan-Meier estimate and $95 \%$ CI for PFS at 3 years was $71 \%$ (54-83\%) and 5 years was $66 \%$ (48-78\%); estimated median PFS was 8.5 years in this subgroup. As reported in the initial manuscript, the majority of relapses occurred distantly. There was one additional recurrence to the liver since the original manuscript, occurring 56 months after study entry. In total there were two local relapses (one of which was both a local and distant failure) that occurred within the radiated field and nine distant recurrences (Fig. 3).

A total of 15 patients (36.6\%) had documented lymphedema in their medical record following treatment. Lymphedema was unilateral in nine patients and bilateral in six patients. The median time from completion of study treatment to patient report of lymphedema was 18.6 months (range 1-60 months). Stage and treatments were not consistently reported. The median time from completion of radiation treatment to patient report of lymphedema was 15 months (range 2.5-62.5 months). The extent of surgery (e.g. performance of both pelvic and para-aortic lymphadenectomy) and the radiation doses were similar between patients who developed lymphedema and those who did not. Five $(33.3 \%)$ of the patients with subsequent lymphedema were treated with extended radiation to the para-aortic chains. No relationship was observed between recurrence, location of recurrence, or surgical approach (laparotomy versus minimally invasive) and risk of subsequent lymphedema. 


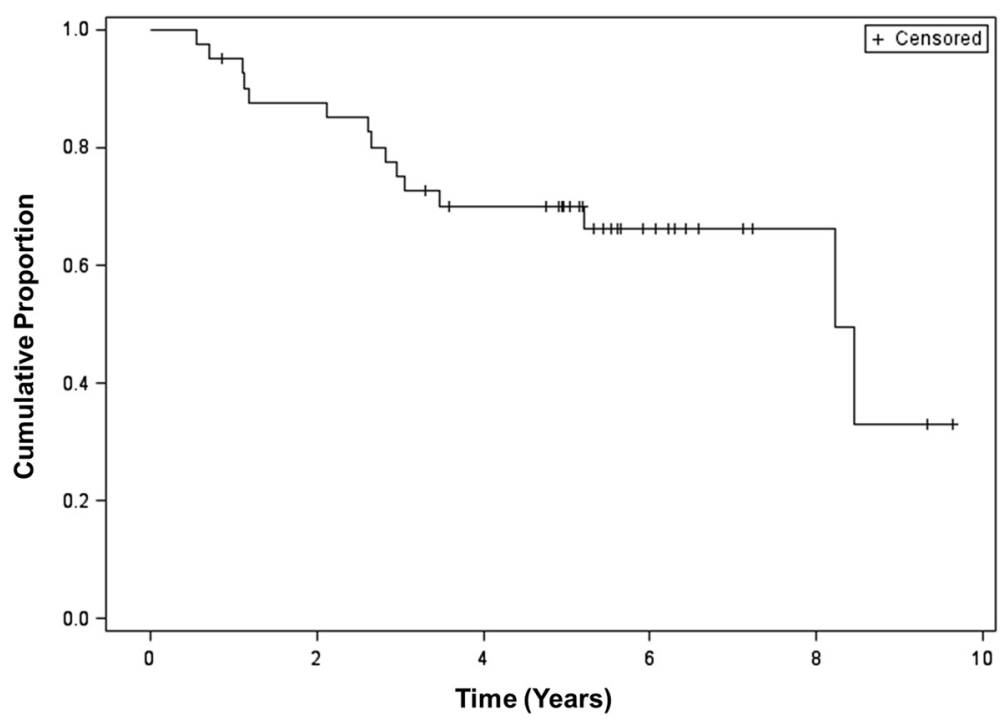

Fig. 1 Overall survival for study participants

\section{Discussion}

This follow-up study of women with advanced stage and recurrent endometrial cancer receiving adjuvant chemotherapy and radiation using the "sandwich" method indicates the treatment demonstrates high long-term efficacy. With a median follow-up of 5 years, estimates of OS and PFS at 5 years remain high at 70 and $66 \%$, respectively. Importantly, there have been no additional pelvic recurrences since the original publication, supporting the use of radiation treatment to prevent local recurrence. There has only been one additional distant recurrence during the follow-up period. This is the longest follow-up data available for this treatment modality to our knowledge.
In this prospective trial we chose to use docetaxel based on the increased neurotoxicity associated with paclitaxel in an endometrial cancer population that often has baseline neuropathy. The activity of docetaxel in endometrial cancer has been previously reported by Katsumata et al. who used docetaxel at $70 \mathrm{mg} / \mathrm{m}^{2}$ every 3 weeks in stage III, IV or recurrent endometrial cancer with an overall response rate of $31 \%$ [13]. Günthert et al. reported response rates of $21 \%$ in a similar previously untreated population [14]. The GOG has conducted a phase II study in previously treated recurrent endometrial cancer patients studying docetaxel $36 \mathrm{mg} / \mathrm{m}^{2}$ administered weekly every 28 days. They reported modest activity with two (7.7 \%)

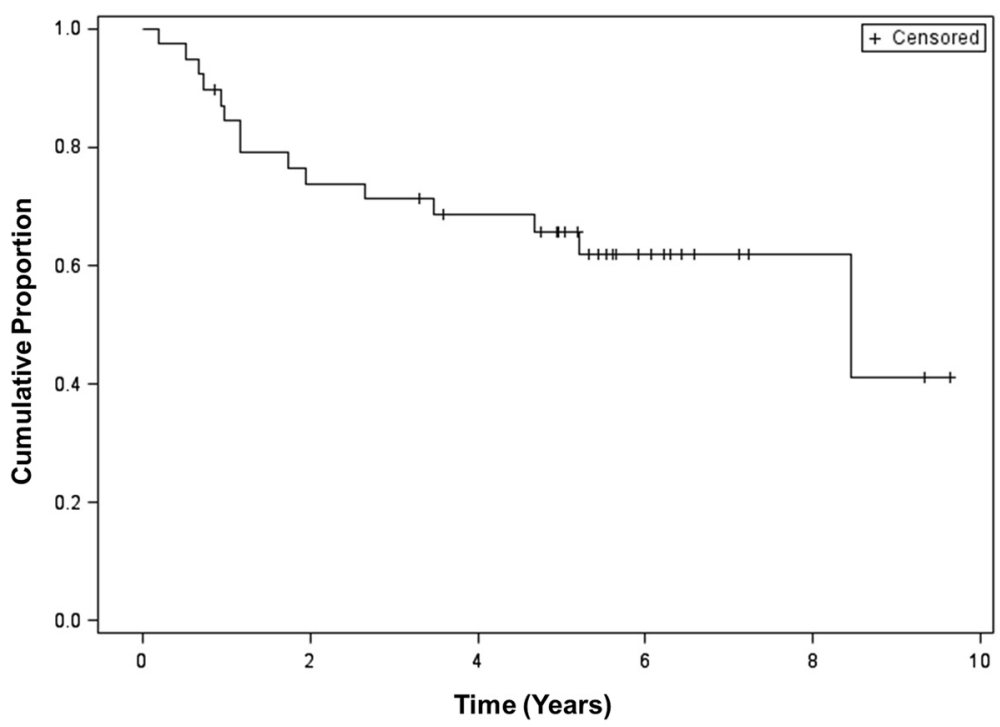

Fig. 2 Progression-free survival for study participants. Excludes two patients who were recurrent at time of study entry 


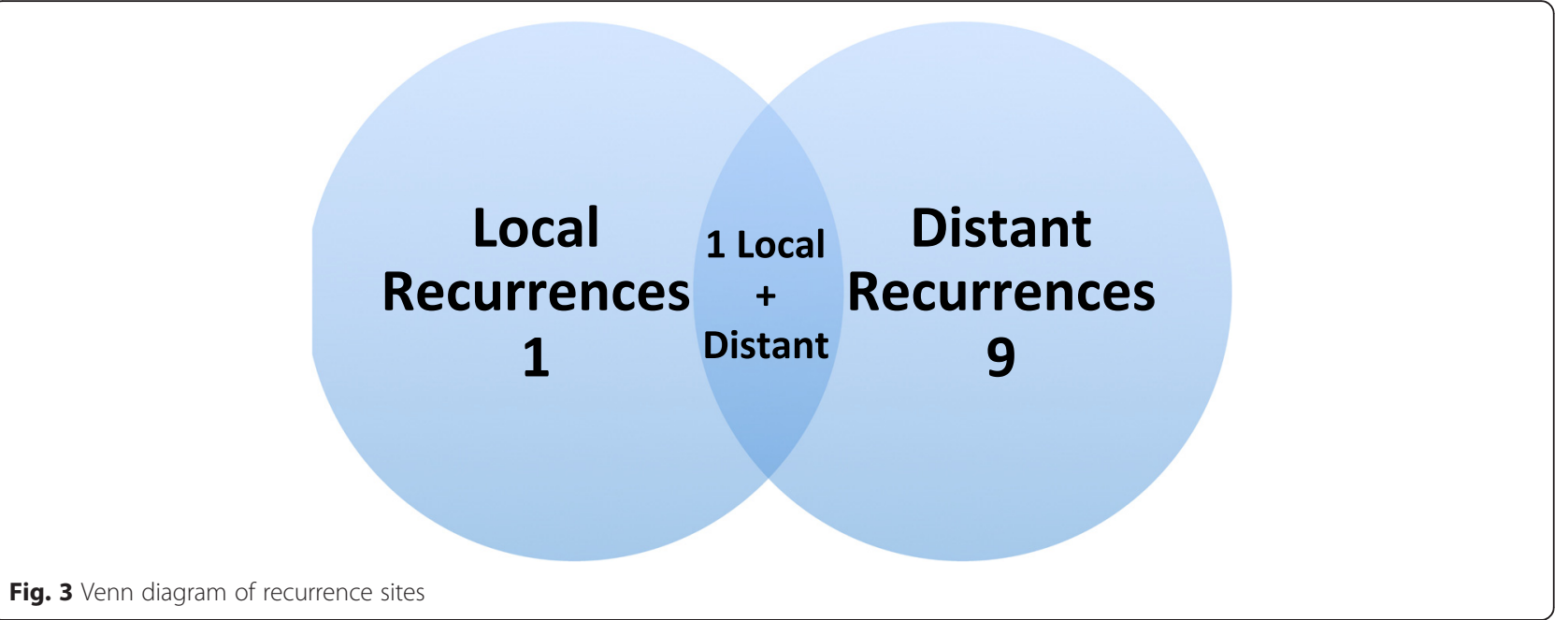

partial responses and eight (30.8 \%) with stable disease [15]. In our study, during treatment only two patients reported grade 2 neuropathy; none experienced grade 3 or 4 neuropathy. Lupe et al. reported that $31 \%$ of their patients receiving paclitaxel and carboplatin interposed by radiation experienced grade 3 or 4 toxicity with peripheral neuropathy and neutropenia being the most commonly cited [16]. The decision to use docetaxel as opposed to paclitaxel and carboplatin over cisplatin is largely due to the grades 3 and 4 neuropathy observed in approximately $40 \%$ of patients receiving cisplatin and paclitaxel. Endometrial cancer patients often are older and many have previouslydiagnosed diabetes, therefore neuropathy tends to be a significant issue in this population. We believed that administration of docetaxel instead of paclitaxel decreased neurotoxicity allowing for completion of six total prescribed courses of chemotherapy.

In GOG 258, the primary objective was to determine if treatment with cisplatin and volume-directed radiation followed by carboplatin and paclitaxel for 4 cycles (experimental arm) reduced the rate of recurrence or death when compared to chemotherapy consisting of carboplatin and paclitaxel for 6 cycles (control arm) in patients with Stages III-IVA endometrial carcinoma. Historically for Stage III endometrial carcinoma, patients underwent surgery followed by radiation therapy. Often, systemic failure beyond treatment fields is an issue in advanced stage endometrial cancer. Alternatively, chemotherapy for this population allows for good systemic control, but poor local control. We choose to study the "sandwich" approach because in theory, sequential rather than concurrent delivery of the two treatment modalities should limit the overall toxicity and allow for maximum therapeutic dosing of both radiation and chemotherapy. Despite thorough surgical staging and cytoreduction, remaining microscopic disease can be present outside the pelvis at the time of adjuvant therapy initiation. Typically following surgery, initiation of therapy can be delayed for 2-3 weeks. If radiation therapy is given initially, as was seen in our patient population we have previously reported, it took a median of 42.5 days (range 34-62) to complete radiation therapy [17].

A multimodality regimen similar to GOG 258 was used in RTOG 9708 [18]. In RTOG 9708, pathologic requirements included grade 2 or 3 endometrial adenocarcinoma with either $>50 \%$ myometrial invasion, cervical stromal invasion, or pelvic-confined extrauterine disease. Patients received 45 Gy in 25 fractions to the pelvis along with cisplatin $(50 \mathrm{mg} / \mathrm{m} 2)$ on days 1 and 28. Vaginal brachytherapy was performed after the external beam radiation. Patients then went on to receive four courses of cisplatin $(50 \mathrm{mg} /$ $\mathrm{m} 2)$ and paclitaxel $(175 \mathrm{mg} / \mathrm{m} 2)$ at 4-week intervals following completion of radiotherapy. Unlike our population that consisted only of advanced stage disease (Stages III and IV) and included serous histology, the patient population in RTOG 9708 represented an earlier stage group (stages I to IIIC); with 39 \% having Stage I or II disease. The inclusion of earlier stage disease may account for the slightly higher 4-year overall survival (OS) and disease-free survival (DFS) of 85 and $81 \%$, respectively. In their stage III patients, the 4-year rates for OS and DFS were 77 and $72 \%$, respectively. In our more advanced stage population, our 4-year rates were OS and DFS was $0.70(0.53-0.82)$ and 0.69 (0.51-0.81), respectively.

As we await the results from GOG 258, the protocol we follow is based on the observation that following radiation therapy there often is a treatment break before beginning chemotherapy. Theoretically, this delay could lead to disease progression prior to beginning systemic therapy in areas outside of the radiation field. Often times, if all six courses of chemotherapy are given followed by tumor directed radiotherapy, patients may have difficulty in finishing the radiation therapy due to toxicity related to the chemotherapy. Administering three cycles of chemotherapy followed by radiation allows for both modalities, with 
the ability to give at least some systemic therapy prior to initiating the radiation phase of the protocol.

In addition to long-term survival, we also examined the rates of lymphedema associated with the "sandwich" method. We found that 15 patients $(36.6 \%)$ were diagnosed with clinically observable lymphedema after treatment. No consistent relationship was observed between the presence of subsequent lymphedema and the extent of surgery, radiation dose, or radiation fields. Many factors may contribute to lymphedema among patients with endometrial cancer, including patient habitus, disease, and treatment-related factors. There is no consensus on the individual weight of these factors in contributing to this treatment toxicity. Several studies have demonstrated that the risk of lymphedema increases with removal of a greater number of lymph nodes. For instance, AbuRustum et al. [10] reported that patients who had ten or more regional lymph nodes removed were at higher risk for developing lymphedema. This association has not been found in all studies [10,19-21]. Additionally, other studies have proposed the risk of lymphedema varies according to which lymph nodes are removed. In opposition, Yost et al. [20] found no difference in lymphedema rates in women with endometrial cancer according to the extent of lymphadenectomy (e.g. pelvic compared with pelvic and paraaortic node dissection) and Todo et al. [19] did not find para-aortic lymphadenectomy to be a risk factor for lymphedema in their review of 286 women with endometrial cancer. Hareyama et al. [22] examined the effect of preserving the circumflex iliac lymph nodes in 329 women with various gynecologic malignancies who underwent both pelvic and para-aortic lymphadenectomies. They found a lower incidence of lymphedema in the patients whose circumflex iliac lymph nodes were not removed. In our study, no significant differences were seen regarding the location of lymphadenectomy (e.g. pelvic versus pelvic and para-aortic) or in the number of lymph nodes removed in women who developed lymphedema.

The literature also suggests that radiation impacts the risk of lymphedema. Todo et al. [19] reported that whole pelvic radiation therapy was an independent risk factor for developing lower-extremity lymphedema, and this risk was reiterated by Yost et al. [20]. This association however, has not been identified in all studies. In a study of 150 women with vulvar cancer there was no increase in the risk of lymphedema in women who had previously received radiation [23]. Ryan et al. reports that in a sample population of women with lower limb lymphedema after gynecologic cancer treatment, more than half of the women with lymphedema reported having to change their daily activities as a result of lymphedema [8]. More attention is now being given to defining the effect lymphadenectomy has on the risk of lymphedema. We await the findings of GOG 244, the LEG study, a prospective longitudinal trial examining the incidence and risk factors for lymphedema and its impact on quality of life in women who have undergone radical gynecologic surgery [24].

The strengths of this study include its ability to report long-term outcomes in patients treated with the "sandwich" method. Additionally, it is the first study to describe the risk of lymphedema with this multimodal treatment. However, given the retrospective approach to identifying post-treatment lymphedema and the loss of follow-up in some patients, it is likely that the reported incidence of lymphedema is underestimated in our study. Additionally, the severity of lymphedema was not always reported, therefore limiting the utility of these data for fully counseling patients on the risk associated with this treatment modality.

\section{Conclusions}

Our study confirms that the "sandwich" approach is a promising treatment for women with advanced and recurrent endometrial cancer, but its subsequent risk of lymphedema is not negligible. It is important to recognize this risk and to counsel patients appropriately, especially as studies have shown that lymphedema can have a significant negative impact on a patient's quality of life after cancer treatment [20]. As the medical community develops better treatment protocols for patients with gynecologic cancers, which will hopefully lead to improved prognoses, it is also important to treat and care for conditions that may result from these cancer treatments. Studies that focus on lymphedema and other long term sequela of treatments in gynecologic cancer patients are necessary to estimate the true prevalence of these unwanted side-effects. We are currently prospectively collecting lymphedema risks and incidence in our endometrial cancer patients being treated in the "sandwich" method. This information will improve our ability to counsel patients on long-term risks of therapy and hopefully, in the future, will inform us on methods of prevention.

\section{Funding source}

The original study was supported financially by Sanofi-Aventis. Statistical data analysis was supported in part by NIH grant P30 CA77598 utilizing the Biostatistics and Bioinformatics Core shared resource of the Masonic Cancer Center, University of Minnesota and by the National Center for Advancing Translational Sciences of the National Institutes of Health Award Number UL1TR000114.

\begin{abstract}
Authors' contributions
MG wrote the introduction and discussion sections and finalized manuscript. RV wrote the initial draft of methods, performed the statistical data analysis and wrote the results sections of the manuscript. JB performed the collection of follow-up data and also conducted a literature review. Both PA and KD were Co-Investigators on the original trial and provided significant input on this manuscript. MA was the Principle Investigator on the original trial, conceived the idea for the follow-up study, and provided significant input on the manuscript All authors read and approved the final manuscript.
\end{abstract}

Competing interests

The authors declare that they have no competing interests. 


\section{Consent section}

Written informed consent was obtained from the patients enrolled in this trial for the publication of the original trial results as well as accompanying images. A copy of the written consent is available for review by the Editor-in-Chief of this journal.

\section{Author details MN 55445, USA. \\ Received: 19 March 2016 Accepted: 19 May 2016 \\ Published online: 26 May 2016}

'Department of Obstetrics, Gynecology and Women's Health, Division of Gynecologic Oncology, University of Minnesota, Minneapolis, MN, USA. ${ }^{2}$ Biostatistics and Bioinformatics Core, Masonic Cancer Center, University of Minnesota, Minneapolis, MN, USA. ${ }^{3}$ Eastern Virginia Medical School, Norfolk, VA, USA. ${ }^{4}$ Radiation Oncology, University of Minnesota, Minneapolis, MN, USA. ${ }^{5}$ University of Minnesota, MMC 395, 420 Delaware St. SE, Minneapolis,

\section{References}

1. Siegel R, Ma J, Zou Z, Jemal A. Cancer statistics, 2014. CA Cancer J Clin. 2014;64:9-29.

2. Amant F, Moerman P, Neven P, Timmerman D, Van Limbergen E, Vergote I. Endometrial cancer. Lancet. 2005;366:491-505.

3. Deleon MC, Ammakkanavar NR, Matei D. Adjuvant therapy for endometrial cancer. J Gynecol Oncol. 2014;25:136-47.

4. Geller MA, Ivy JJ, Ghebre R, Downs Jr LS, Judson PL, Carson LF, et al. A phase II trial of carboplatin and docetaxel followed by radiotherapy given in a "Sandwich" method for stage III, IV, and recurrent endometrial cancer. Gynecol Oncol. 2011;121:112-7.

5. Abaid LN, Rettenmaier MA, Brown 3rd JV, Micha JP, Mendivil AA, Wabe MA, et al. Sequential chemotherapy and radiotherapy as sandwich therapy for the treatment of high risk endometrial cancer. J Gynecol Oncol. 2012;23:22-7.

6. Einstein MH, Frimer M, Kuo DY, Reimers LL, Mehta K, Mutyala S, et al. Phase II trial of adjuvant pelvic radiation "sandwiched" between combination paclitaxel and carboplatin in women with uterine papillary serous carcinoma. Gynecol Oncol. 2012;124:21-5.

7. Dogan NU, Yavas G, Yavas C, Ata O, Yilmaz SA, Celik C. Comparison of "sandwich chemo-radiotherapy" and six cycles of chemotherapy followed by adjuvant radiotherapy in patients with stage IIIC endometrial cancer: a single center experience. Arch Gynecol Obstet. 2013;288:845-50.

8. Ryan M, Stainton MC, Jaconelli C, Watts S, Mackenzie P, Mansberg T. The experience of lower limb lymphedema for women after treatment for gynecologic cancer. Oncol Nurs Forum. 2003;30:417-23.

9. Beesley VL, Rowlands IJ, Hayes SC, Janda M, O'Rourke P, Marquart L, et al. Incidence, risk factors and estimates of a woman's risk of developing secondary lower limb lymphedema and lymphedema-specific supportive care needs in women treated for endometrial cancer. Gynecol Oncol. 2015;136:87-93.

10. Abu-Rustum NR, Alektiar K, lasonos A, Lev G, Sonoda Y, Aghajanian C, et al. The incidence of symptomatic lower-extremity lymphedema following treatment of uterine corpus malignancies: a 12-year experience at Memorial Sloan-Kettering Cancer Center. Gynecol Oncol. 2006;103:714-8.

11. Ghezzi F, Uccella S, Cromi A, Bogani G, Robba C, Serati M, et al. Lymphoceles, lymphorrhea, and lymphedema after laparoscopic and open endometrial cancer staging. Ann Surg Oncol. 2012:19:259-67.

12. Kaplan $E$, Meier $P$. Nonparametric estimation from incomplete observations. J Am Stat Assoc. 1958:53:457-81.

13. Katsumata N, Noda K, Nozawa S, Kitagawa R, Nishimura R, Yamaguchi S, et al. Phase II trial of docetaxel in advanced or metastatic endometrial cancer: a Japanese Cooperative Study. Br J Cancer. 2005;93:999-1004.

14. Gunthert AR, Ackermann S, Beckmann MW, Camara O, Kiesel L, Rensing K, et al. Phase II study of weekly docetaxel in patients with recurrent or metastatic endometrial cancer: AGO Uterus-4. Gynecol Oncol. 2007;104:86-90.

15. Garcia AA, Blessing JA, Nolte S, Mannel RS. A phase li evaluation of weekly docetaxel in the treatment of recurrent or persistent endometrial carcinoma: a study by the Gynecologic Onclogy Group. Gynecol Oncol. 2008:111:22-6.

16. Lupe K, D'Souza DP, Kwon JS, Radwan JS, Harle IA, Hammond JA, et al. Adjuvant carboplatin and paclitaxel chemotherapy interposed with involved field radiation for advanced endometrial cancer. Gynecol Oncol. 2009;114:94-8.

17. Geller MA, Ivy J, Dusenbery KE, Ghebre R, Isaksson Vogel R, Argenta PA. A single institution experience using sequential multi-modality adjuvant chemotherapy and radiation in the "sandwich" method for high risk endoemtrial carcinoma. Gynecol Oncol. 2010:118:19-23.

18. Greven K, Winter K, Underhill K, Fontenesci J, Cooper, Burke T. Final analysis of RTOG 9708: adjuvant postoperative irradiation combined with cisplatin/ paclitaxel chemotherapu following surgery for patients with high-risk endometrial cancer. Gynecol Oncol. 2006;103:155-9.

19. Todo Y, Yamamoto R, Minobe S, Suzuki Y, Takeshi U, Nakatani M, et al. Risk factors for postoperative lower-extremity lymphedema in endometrial cancer survivors who had treatment including lymphadenectomy. Gynecol Oncol. 2010;119:60-4.

20. Yost KJ, Cheville AL, Al-Hilli MM, Mariani A, Barrette BA, McGree ME, et al. Lymphedema after surgery for endometrial cancer: prevalence, risk factors, and quality of life. Obstet Gynecol. 2014;124:307-15.

21. Tada H, Teramukai S, Fukushima M, Sasaki H. Risk factors for lower limb lymphedema after lymph node dissection in patients with ovarian and uterine carcinoma. BMC Cancer. 2009;9:47.

22. Hareyama H, Ito K, Hada K, Uchida A, Hayakashi Y, Hirayama E, et al. Reduction/prevention of lower extremity lymphedema after pelvic and para-aortic lymphadenectomy for patients with gynecologic malignancies. Ann Surg Oncol. 2012;19:268-73.

23. Carlson JW, Kauderer J, Walker JL, Gold MA, O'Malley D, Tuller E, et al. A randomized phase III trial of VH fibrin sealant to reduce lymphedema after inguinal lymph node dissection: a Gynecologic Oncology Group study. Gynecol Oncol. 2008;110:76-82.

24. Gynecologic Oncology Group. The Lymphedema and Gynecologic Cancer (LEG) Study: Incidence, Risk Factors, and Impact in Newly Diagnosed Patients. Bethesda: ClinicalTrials.gov; 2014.

25. Gehrig PA, Boggess JF, Moller KA, Boruta DM, Dunlap AP, Fowler WC, et al. Paclitaxel and carboplatin sequenced with radiotherapy in women with advanced uterine serous carcinoma: is this optimal therapy? J Clin Oncol. 2004:22:5084.

26. Lupe K, Kwon J, D'Souza D, Gawlik C, Stitt L, Whiston F, et al. Adjuvant paclitaxel and carboplatin chemotherapy with involved field radiation in advanced endometrial cancer: a sequential approach. Int. J Radiat Oncol Biol Phys. 2007;67:110-116.

27. Alvarez Secord A, Havrilesky LJ, Bae-Jump V, Chin J, Calingaert B, Bland A, et al. The role of multi-multimodality adjuvant chemotherapy and radiation in women with advanced stage endometrial cancer. Gynecol Oncol. 2007;107: 285-291.

28. Fields AL, Einstein MH, Novetsky AP, Gebb J, Goldberg GL. Pilot phase II trial of radiation "sandwiched" between combination paclitaxel/platinum chemotherapy in patients with uterine papillary serous carcinoma (UPSC). Gynecol Oncol. 2008;108:201-206.

29. Secord AA, Havrilesky L, O'Malley DM, Bae-Jump V, Fleming ND, Broadwater $G$, et al. A multicenter evaluation of sequential multimodality therapy and clinical outcome for the treatment of advanced endometrial cancer. Gynecol Oncol. 2009;114:442-447.

30. Lan $\mathrm{C}$, Huang $X$, Cao $X$, Huang $H$, Feng $Y$, Huang $Y$, et al. Adjuvant docetaxel and carboplatin chemotherapy administered alone or with radiotherapy in a "sandwich" protocol in patients with advanced endometrial cancer: a single-institution experience. Expert Opin Pharmacother. 2013;14:535-42.

\section{Submit your next manuscript to BioMed Central and we will help you at every step:}

- We accept pre-submission inquiries

- Our selector tool helps you to find the most relevant journal

- We provide round the clock customer support

- Convenient online submission

- Thorough peer review

- Inclusion in PubMed and all major indexing services

- Maximum visibility for your research

Submit your manuscript at www.biomedcentral.com/submit 